\title{
The effect of non-informative spatial sounds on haptic scene recognition
}

\author{
Jason S. Chan and Fiona N. Newell ${ }^{*}$ \\ School of Psychology and Institute of Neuroscience, \\ Lloyd Building, \\ Trinity College, Dublin 2, Ireland \\ Fax: +35316712006 \\ E-mail: jchan@tcd.ie \\ E-mail: fnewell@tcd.ie \\ *Corresponding author
}

\begin{abstract}
Previous studies found that performance in tactile or haptic spatial tasks improved when non-informative visual information was available, suggesting that vision provides a precise spatial frame to which tactile information is referred. Here, we explored whether another intrinsically spatial modality, audition, can also affect haptic recognition. In all experiments, blindfolded participants first learned a scene through touch and were subsequently required to recognise the scene. We found no effect on haptic performance when white noise stimuli were presented from specific locations (Experiment 1). However, performance was significantly reduced by pure tone stimuli presented from the same locations (Experiment 2), moreover, these tones disrupted recall but not encoding of the haptic scene (Experiment 3). In Experiment 4, we found that spatial rather than non-spatial auditory information was required to affect haptic performance. Finally, in Experiment 5 we found no specific benefit for familiar sound cues over unfamiliar or no sounds on haptic spatial performance. Our findings suggest that, in contrast to vision, auditory information is unlikely to have sufficient spatial precision therefore disrupting the spatial representation of haptic information. Our results add to a growing body of evidence for multisensory influences in the perception of space.
\end{abstract}

Keywords: audition; haptics; scene recognition; non-informative; multisensory.

Reference to this paper should be made as follows: Chan, J.S. and Newell, F.N. (2013) 'The effect of non-informative spatial sounds on haptic scene recognition', Int. J. Autonomous and Adaptive Communications Systems, Vol. 6, No. 4, pp.342-365.

Biographical notes: Jason S. Chan earned his $\mathrm{PhD}$ in Experimental Psychology from Oxford University in 2002. Currently, he is a Postdoctoral Research Fellow in the Institute of Neuroscience at Trinity College Dublin, but will move to the Institute of Medical Psychology at Goethe University in early 2011. His primary focus of research is multisensory perception, with an emphasis on spatial perception. He is skilled in behavioural as well as fMRI neuroimaging research design and analyses. He has been awarded several prestigious grants, such as the postdoctoral fellowship from the Irish Research Council for the Humanities and Social Sciences. 
Fiona N. Newell obtained her PhD from the University of Durham, UK. In 2000, she took up a Lectureship position in the School of Psychology, Trinity College, Dublin. Her main research interests are in human sensory and perceptual processes. The main goal of her research is to provide a better understanding of how information is shared across the senses and to elucidate the brain processes involved in the perception of objects, faces and places across the main human sensory systems. She has published papers in many of the leading journals and her research is funded by several national and international agencies.

\section{Introduction}

In typical investigations on spatial perception, efforts are made to control for as many external, within-modal variables as possible that may interfere with spatial processing in that modality. However, these investigations often fail to mention whether the same amount of effort is applied to control the effect of stimuli from other modalities, particularly, those that are considered irrelevant to the immediate task. This is because it was assumed that spatial information from task-irrelevant modalities was unlikely to have any effect on performance. In contrast, the results of a few recent studies suggest that this may be an erroneous assumption as spatial information from another modality, even if that information is irrelevant to the spatial task at hand, can influence spatial performance.

Several recent studies have demonstrated the effect of so-called 'non-informative' or task-irrelevant visual information on spatial processing in the tactile modality. Initial reports suggested that visual information can affect somatosensory representations of the body (e.g. Kennett et al., 2001; Press et al., 2004). For example, Kennett et al. found that somatosensory two-point discrimination thresholds were reduced when participants could view their arm prior to tactile stimulation. Remarkably, when the participants viewed a magnified image of their arm, thresholds for the two-point task further decreased. In contrast, thresholds increased when the image of their arm appeared unnaturally small. Later, Press et al. (2004) suggested that the effects of non-informative vision may be limited to more difficult, cross-modal spatial tasks and may not be observed for relatively easy tasks. These visual effects on somatosensory processing are thought to be mediated by the receptive fields of body parts in somatosensory cortex overlapping with visual receptive fields when the same body part is viewed (Graziano and Gross, 1993; see Holmes and Spence, 2004 for a review; Rizzolatti et al., 1997; Schicke, 2007). Using electroencephalography, Taylor-Clark et al. (2002) reported evidence that neuronal processing in the somatosensory areas, SI and SII, of the brain was affected by visual stimulation. In particular, they found greater N80 and N180 responses in the somatosensory cortex when participants were able to see their arm. Taylor-Clarke et al. suggested that this was evidence of a feedback loop from the posterior parietal area to areas SI and SII, leading to enhanced activation of the somatosensory areas and, consequently, a modulation in perception (see also Haggard et al., 2007). Thus changes in somatosensory precision demonstrate vision's role as a scaling measure for the tactile receptive field (Pears and Jackson, 2004). 
Other studies investigated the role of vision on the spatial perception of objects external to the body which were explored through touch (e.g. Newport et al., 2002; Zuidhoek et al., 2004). For example, Newport et al. (2002) examined the effects of irrelevant, or so-called 'non-informative', visuo-spatial information on a haptic object orientation matching task. Without seeing the stimuli, participants were required to rotate a test bar with one hand until it was parallel to a reference bar held by their other hand. Performance in this task was best when participants could view their surroundings (but not the stimuli) than when they were blindfolded. However, this benefit for non-informative vision occurred only when the task favoured an extrinsic over an intrinsic coding of tactile space, suggesting that vision provides an external reference frame to which spatial events in other modalities are referred. In a later study, Zuidhoek et al. (2004) investigated the nature of this reference frame further by pitting attentional orienting against the availability of non-informative visual cues. They found that regardless of head or eye orientation, visual information improved the haptic task. However, orienting towards the reference bar facilitated performance in both the non-informative visual and blindfolded tasks. This benefit for orientating towards the reference bar may have been mediated by mental imagery of the reference bar. Furthermore, orientating to that area of space, even though it is not visible to the participant, may nevertheless have aided in the allocation of attentional resources (Driver and Grossenbacher, 1996; Lloyd et al., 2003; Spence and Driver, 1996; Zuidhoek et al., 2004) and possibly reduce perceptual load (Lavie, 1995; Lavie and Tsal, 1994). In sum, as Zuidhoek et al. argue, the overall findings from this study suggest that the effects of attention and non-informative vision are independent such that noninformative vision can influence haptic task performance regardless of attentional orienting.

In a recent study, Pasqualotto et al. (submitted) confirmed that non-informative vision can enhance performance in a haptic spatial memory task where participants were required to learn and later recognise the location of a set of objects arranged in a scene using touch only. Haptic spatial recognition performance was enhanced when participants could view their surroundings than when they were blindfolded.

Although, as indicated above, many studies have demonstrated the effect of visual information on tactile spatial perception, to date, the effect of non-informative audition on haptic spatial perception has not been investigated. The visual modality is thought to dominate spatial perception because of its relatively higher spatial acuity, in contrast to the auditory modality which is thought to dominate temporal processing in other modalities (Colavita, 1974). However, some studies have suggested that audition can affect spatial perception in either visual discrimination tasks (Arnott and Goodale, 2006) or large-scale navigation tasks (Klatzky et al., 2003, 2006). For example, Arnott and Goodale found that presenting a sound to the left or right of a visually presented vernier display elicited an 'attentional repulsion' effect whereby the visual stimulus was perceived to have moved horizontally in the opposite direction to the auditory sound. If visual space can be distorted by sound then it is possible that sound can be used to influence haptic space and, consequently, haptic spatial memory.

Some studies have found that auditory stimuli are as reliable a source of spatial locations as visual stimuli. For example, in an experiment conducted by Klatzky et al. (2002), participants were either presented with a visual or auditory cue at various azimuths and their task was to turn a handle to indicate the correct angle of the location 
of the cue. They found no significant difference in performance between visual and auditory cued locations. However, in another study Loomis et al. (1998) compared visual and auditory spatial localisation of stimuli presented across different azimuth as well as distant locations. Participants walked a direct or indirect path towards either a visual or auditory target. Participants were able to reliably estimate the location of a visual target by walking, however, there was a systematic underestimation of distance to an auditory target. Later, Klatzky et al. (2003) found that spatial updating was best for visual over auditory cues, although performance to 3D sounds was better than spatial updating via language cues. Overall, these data suggest that although auditory location at azimuth is relatively accurate, spatial differences between vision and audition can emerge when targets are presented at different distances in depth.

Although both vision and audition carry information about the spatial location of objects, the extent to which auditory information affects performance in a tactile task was hitherto relatively unexplored. Some studies have suggested that information about spatial audition and touch can interact to affect performance. For example, Caclin et al. (2002) found that in an audio-tactile analogue of the ventriloquist effect, participants mislocalised the source of a tone when it was presented synchronously with an incongruously located tactile vibration. This shows a clear cross-modal interaction between audition and touch for spatial perception.

In the following study, we investigated if auditory stimuli that are non-informative to a haptic task, affect recognition performance for the spatial locations of a scene of objects encoded through touch. We used a similar haptic apparatus and design to that described in Pasqualotto et al. (2005). In all experiments, participants were required to learn the location of a set of objects arranged in a scene using touch only. They were, subsequently, tested on their recognition of the relative locations of these objects. Participants were blindfolded throughout these tasks and across all five experiments we manipulated auditory information such that participants either received non-informative sounds during the haptic task or no sounds were heard. We hypothesised that these spatial sounds, even though irrelevant to the task, would affect haptic performance. We expected, given that audition is an intrinsically spatial modality, that the presence of auditory cues would enhance haptic performance by providing an external reference frame to which the haptic locations of the objects could be encoded. However, since some auditory information can be less reliable than vision for efficient spatial localisation (e.g. see Arnott and Goodale, 2006; Klatzky et al., 2003) it was also possible that the effect of auditory cues on haptic spatial performance may be dependent on the nature of the auditory cue.

\section{General methodology}

\subsection{Apparatus and materials}

The object stimuli consisted of a set of 16 toy objects representing human characters and common animals (e.g. cow, sheep and rooster). All of these objects were $1-\mathrm{cm}$ wide, varied in height from the base to the top of the object between 6 and $8 \mathrm{~cm}$ and varied in length from 3.5 to $5.5 \mathrm{~cm}$. Prior to the experiment, we established that these stimuli were readily distinguishable from each other through touch alone. For any given trial, seven objects were randomly selected from the entire set and placed on a 
$(57 \mathrm{~cm})$ circular platform in random positions (the haptic platform and stimuli described by Pasqualotto etal. (2005) were used here). The platform had 19 sunken position markers in which the individual objects could be randomly placed. Each position marker was equidistant from any of its neighbouring markers by a distance of $7 \mathrm{~cm}$. The platform was positioned on a table which was placed directly in front of the participant.

The experiments were conducted in a sound attenuated laboratory in the School of Psychology, Trinity College Dublin. Prior to testing, we insured that this room was unfamiliar to all participants in our experiments. The laboratory measured $4.5 \mathrm{~m} \times 2.8 \mathrm{~m}$. A table, on which the haptic apparatus was placed, was positioned in the middle of this room. Four loudspeakers were positioned in the room such that each one was placed at each corner of the room. Each loudspeaker (Visaton, model: FRWS 5) measured $4.5 \mathrm{~cm}$ and was mounted on a stand which measured $1.13 \mathrm{~m}$ from the ground. Each of the four corner stands was positioned at a distance of $2.24 \mathrm{~m}$ from the participant (see Figure 1 for details). The onset and offset of the auditory stimuli were controlled by a desktop PC using a custom built program.

Figure 1 A bird's eye illustration of the haptic apparatus and experimental room (see online version for colours)

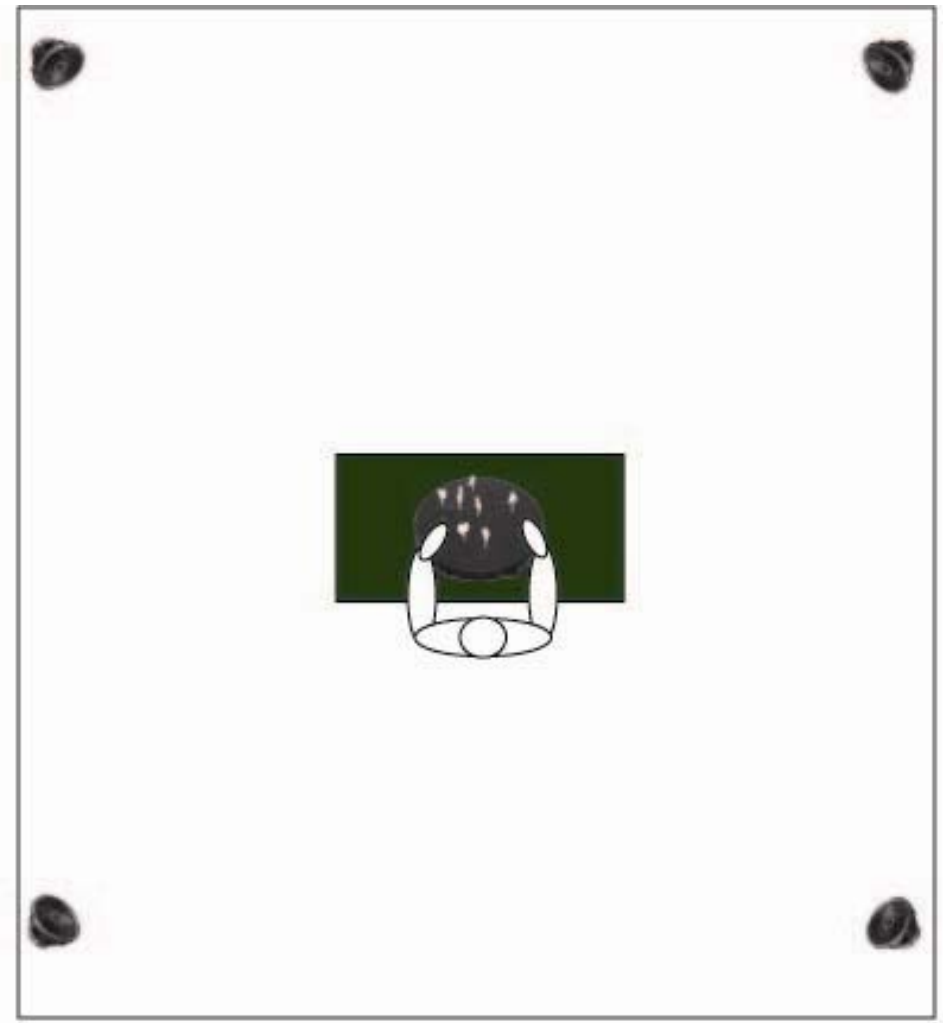

Note: The participant and haptic apparatus were positioned in the middle of the testing room. Four loudspeakers were positioned in each corner of the testing room at equidistance from the position of the participant at a distance of $2.24 \mathrm{~m}$. 


\subsection{Procedure}

Participants were instructed about their task prior to entering the experimental room. This was done to minimise any ambient sound that might give the participants a sense of the room's spatial dimensions prior to the task. Participants were then blindfolded before entering the experimental room and were guided inside the room by the experimenter to their seat. They then positioned the haptic platform in such a way that they could comfortably reach the far side of the platform.

In all our experiments, a trial consisted of 1-min learning of the seven objects and their positions in the scene followed by a recognition test which terminated when the participant responded (i.e. there was no time limit imposed during the testing session, although participants were encouraged to provide an answer if more than $2 \mathrm{~min}$ had elapsed). No explicit instructions were provided for exploring the scene of objects and participants used both hands to explore the scene. After the 1-min learning session, participants removed their hands from the platform. The experimenter then randomly chose two of the seven objects in the scene and exchanged their locations (i.e. the global configuration of the object locations remained the same). For half of the trials the scene was rotated by the experimenter between learning and test. Sounds were not presented during this interval. After a 20 -sec interval, the test began and participants were required to indicate which two of the seven objects in the scene had swapped locations during the interval. The participants provided their response by pointing to the two objects they thought had changed location. Correct responses were recorded by the experimenter in the following manner: if both objects were correctly identified the score was $100 \%$ correct; if only one object was correct the score was $50 \%$ and if none were correct then the score was $0 \%$.

To promote the use of a more allocentric or extrinsic reference frame to encode the position of objects (Newport et al., 2002; Simons et al., 2002; Zuidhoek et al., 2004) in half of the trials, the entire scene was rotated by $60^{\circ}$ in a clockwise direction relative to the participant between the learning and test sessions. In the remaining half of trials, the scene was not rotated between the learning and test (although the scene was rotated and returned to the original position in case participants were using the sound of the rotating platform as a cue to prepare for the rotation). Participants were generally aware that the platform could be rotated for some trials in the experiment but did not know for which specific trials.

Our protocol was approved by the School of Psychology Research Ethics Committee and all participants provided informed written consent to take part. None of our participants were involved in more than one of the experiments reported here.

\section{Experiment 1}

In the following experiment, whilst participants conducted the haptic object location task we presented a set of white noise bursts across four successive locations (i.e. the four corners of the laboratory). Due to its auditory complexity, white noise is relatively easier to locate than other sounds, e.g. pure tones (Blauert, 1997). To make the auditory spatial information relatively uncomplicated, the order of the locations of these sounds was constrained such that each sound followed another in a clockwise manner around the room. We predicted that if these sounds provided an intrinsic, spatial reference frame 
then we should observe the same benefits on haptic spatial object performance as was found with non-informative visual information (e.g. Newport et al., 2002; Pasqualotto et al., submitted).

\subsection{Method}

\subsubsection{Participants}

Forty students from Trinity College Dublin $($ female $=24$, male $=16$ ) between the ages of 18 and 36 (mean age $=24.33$ years), took part in this experiment for either research credits or pay ( $€ 10$ per hour). All but four participants reported to be right-hand dominant. None of the participants reported any tactile or auditory impairment.

\subsubsection{Auditory stimuli}

The general apparatus is described above. An auditory stimulus consisted of a 1-sec burst of white noise (rise and decay time of $5 \mathrm{~ms}$ ) and the sound pressure level (SPL) was $72 \mathrm{dBA}$ relative to the participant. Stimuli were presented in succession from each of the four loudspeakers in order: the first stimulus was emitted from the rear-left loudspeaker in the room (relative to the participant), the second was emitted from the front-left loudspeaker and so on in a clockwise fashion around the room. There was no delay between the offset of one sound and the onset of the next. These auditory stimuli were presented throughout both the learning and test phases of a trial, according to the design of the experiment.

\subsubsection{Design}

The experiment was based on a mixed design, with audition (non-informative sounds or no sounds) as the between-subjects factor and scene rotation (same or rotated) as the within-subjects factor. Participants were randomly assigned to one of the auditory conditions. Trials during which the scene was rotated were randomly presented amongst trials where the scene was presented in the same position during learning and test.

\subsubsection{Procedure}

Participants randomly allocated to the 'non-informative sound' condition were instructed to ignore the auditory stimuli during the haptic task. For the learning session in the trial, participants began to explore the objects using touch as soon as they heard the first auditory stimulus. After $1 \mathrm{~min}$ of haptic exploration, they were instructed to remove their hands from the scene. In the test period, once again participants began to explore the scene as soon as they heard the first auditory stimulus. The auditory sequence of stimuli was stopped by the experimenter as soon as the participant responded. In the 'no-sound' condition, the experimenter indicated to the participant when to start and stop exploring the scene during learning using the same timing procedures as those used for the participants allocated to the 'non-informative sound' condition. 
There were 12 trials presented during the experiment. In half of these trials, the entire platform was rotated $60^{\circ}$ clockwise (relative to the participant) between learning and test. Two practice trials were presented to the participants before starting the experiment. The experiment lasted approximately $1 \mathrm{hr}$ for each participant.

\subsection{Results}

The percentage correct responses across participant groups and scene rotation conditions are plotted in Figure 2. A mixed analysis of variance (ANOVA) was conducted on the percentage correct responses with audition (no sound or non-informative sounds) as the between groups factor and scene rotation (same or rotated) as a within-subjects factor. We found no main effect of audition $[F(1,33)<1$, ns]. There was a significant main effect of scene rotation $[F(1,33)=8.31, p<0.01]$ : participants were more accurate at identifying the objects which had swapped positions when the scene was not rotated than when it was. There was no evidence of an interaction between the sound and scene rotation conditions $[F(1,33)<1, \mathrm{~ns}]$.

Figure 2 Plot showing the mean percent correct responses in the haptic scene recognition task in Experiment 1 for each of the non-informative sound (i.e. white noise stimuli) and no sound conditions

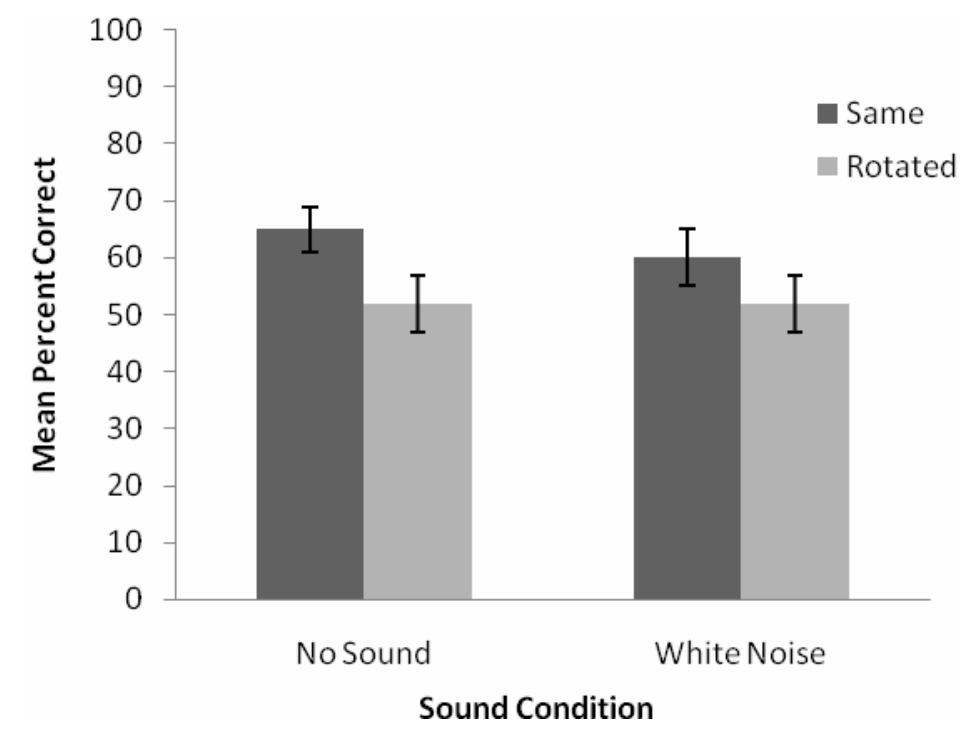

Note: The error bars represent \pm 1 SEM. Here, as in all the experiments, chance level performance is calculated as $(2 / 7 \times 1 / 6=) 4.76 \%$ and performance in all conditions is above this level. 


\subsection{Discussion}

In this experiment, we found no benefit of non-informative sounds (i.e. white noise bursts) on performance in a haptic scene recognition task. This finding is contrary to previous reports that non-informative vision benefits perception in the tactile modality (e.g. Kennett et al., 2001; Newport et al., 2002; Press et al., 2004). Our results suggest that audition is not a sufficiently reliable source of spatial information to affect performance in the tactile modality. However, before such a conclusion can be asserted, we noticed that there were differences in the nature of the auditory information provided in our study compared to the visual information available in other studies. Firstly, here we presented a display of homogenous auditory stimuli (i.e. all white noise bursts presented at the same decibel), whereas previous reports of non-informative vision suggest that the surrounding visual stimuli were heterogeneous landmark objects (e.g. items of furniture present in the room). Thus variation in the nature of the stimuli presented in the non-informative modality may be required in order to reliably spatially locate objects encoded through another modality. Secondly, since we presented the same auditory stimulus in successive locations, participants may have had the impression that the auditory stimulus was moving throughout the room, thus making the auditory stimuli spatially unreliable. Indeed, during our debriefing sessions some participants indicated that this was what was perceived. In the following experiment, we aimed to address these concerns by presenting heterogeneous auditory stimuli (i.e. pure tones each with a different frequency) from each of the original four loudspeakers.

\section{Experiment 2}

In this experiment, each spatial position in the room was marked by a specific auditory sound, which is a set-up that is more similar to those described in studies involving noninformative visual information. Again, we used the same four spatial positions of loudspeakers that were used in the previous experiment and sounds were emitted across successive spatial locations. Thus the only difference between this experiment and the previous experiment was on the nature of the sounds presented, and not the location or the order of the sounds.

\subsection{Method}

\subsubsection{Participants}

Thirteen students from Trinity College Dublin (female $=11$, male $=2$ ) participated in this experiment for either research credits or pay. Participants were aged between 18 and 37 year (mean age $=20.92$ ). All but three of the participants reported to be right-hand dominant. None of the participants reported any tactile or auditory impairment.

\subsubsection{Auditory stimuli}

Unlike in the previous experiment, here the auditory stimuli were tones of varying frequencies: a different pure tone was presented for $1 \mathrm{sec}$ from each loudspeaker 
positioned in each corner of the experimental room. The tones were arranged across the loudspeakers as follows: with respect to the position of the participant, an $800 \mathrm{~Hz}$ tone was presented from the rear-left corner, a $400 \mathrm{~Hz}$ tone was presented from the front-left corner, a $600 \mathrm{~Hz}$ tone was presented from front-right corner and a $1,000 \mathrm{~Hz}$ tone was presented from the rear-right corner. The SPL for each sound was $72 \mathrm{dBA}$ relative to the participant (i.e. same as Experiment 1). This pattern of presentation was adopted to minimise the auditory perception of increasing height (Blauert, 1968, 1997). All pure tones were enveloped, with a $5 \mathrm{~ms}$ rise and decay time.

\subsubsection{Design and procedure}

The experiment was again based on a mixed design with audition (non-informative sounds or no sounds) as the between-subjects factor and scene rotation as the withinsubjects factor. Participants in this experiment were all allocated to the 'sound' condition only and we compared their performance to the participants allocated to the 'no sound' condition in the previous experiment. In all other respects, the design and procedure were the same as described in Experiment 1.

\subsection{Results}

The percentage correct responses across the sound and scene rotation conditions are shown in Figure 3. For convenience, the data from the present experiment are plotted with data from the participants in the "no sound' condition from the previous experiment. We conducted a $2 \times 2$ mixed ANOVA with audition (non-informative sound or no sound) as a between-subjects factor and scene rotation (rotated or not rotated) as within-subjects factor. We found a significant main effect of non-informative audition $[F(1,31)=4.71$, $p<0.038$ ] which was due to significantly better performance in the 'no sound' than in the 'non-informative sound' condition. There was also a main effect of scene rotation $[F(1,31)=10.14, p=0.003]$ with better performance when the scene was presented in the same orientation than when it was rotated between learning and test. There was no interaction between the two main factors $[F(1,31)<1$, ns $]$.

\subsection{Discussion}

We found that the presence of heterogeneous auditory stimuli, although each was consistently located in the same spatial positions, reduced performance in the haptic scene recognition task compared to when no auditory spatial locations were provided. Also, the presence of varying auditory cues disrupted haptic spatial performance relative to non-varying auditory cues as presented in Experiment 1 (since we found no difference between the non-varying sound cues and the no sound cue in Experiment 1). One suggestion for this disruption in performance may have been that the auditory spatial reference cues were mislocalised if their specific location was difficult to determine, as may be the case for the pure tones in particular (Blauert, 1997). This, in turn, may have led to a distortion of auditory space (Arnott and Goodale, 2006) and, consequently, unreliable extrinsic reference frame to which the haptic spatial locations of objects may have been encoded. 
Figure 3 Plot showing the mean percent correct responses for the haptic scene recognition task in Experiment 2

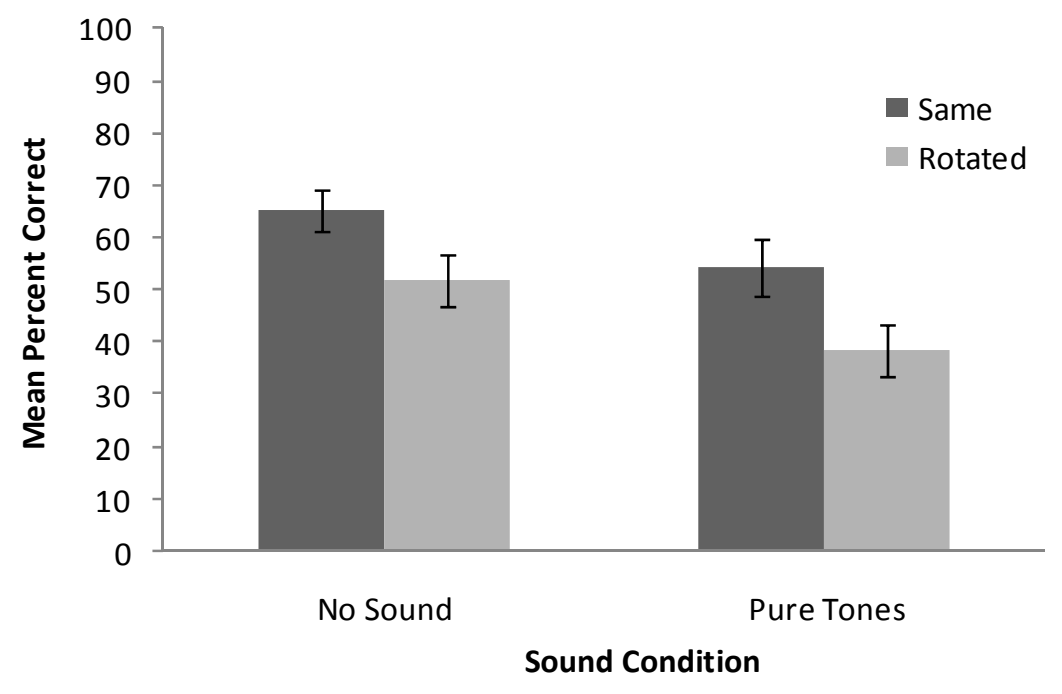

Note: In this experiment, participants received pure tone stimuli in the non-informative sound condition. Data from the 'no sound' condition of Experiment 1 are inserted for comparative purposes. The error bars represent \pm 1 SEM.

It is interesting to note that we found a main effect of scene rotation on performance in both Experiments 1 and 2 and that, more importantly, scene rotation did not interact with the sound conditions. In other words, although the presence of sounds disrupted overall performance in the present experiment it did not incur a further cost on performance when the scene was rotated. This finding suggests that the nature of the representation of the scene was the same across the no sound and sound conditions but that the presence of sounds introduced a general uncertainty into the memory representation of the haptic spatial scene.

In order to assess the effectiveness of our auditory stimuli as spatial cues, we conducted a brief sound localisation experiment using the white noise bursts from Experiment 1 and the different tones used in Experiment 2 within the same testing room. Although it is known that the spatial localisation of white noise is better than the localisation of pure tones (Blauert, 1997; Jeffress, 1972; Trahiotis and Stern, 1989) it was important for us to assess the specific accuracy of each of these sound types in our environment in order to elucidate our effects and provide a context for the following experiments. Five students from the School of Psychology participated in this task for research credits. Each participant was blindfolded before entering the room and, once inside, was guided to the seating position in the centre of the room. They were instructed to simply point to the location of an auditory stimulus, using a laser pointer, as soon as it was heard. The loudspeakers were located in the same positions as Experiments 1 and 2. The experimenter then took note of the perceived location of the sound source for each participant. We found that the relative localisation of the white noise stimuli used in Experiment 1 was better (mean distance error $=53.36 \mathrm{~cm}$ ) than the localisation of the pure tones (mean distance error $=187.90 \mathrm{~cm}$ ). Thus the accuracy at detecting the location 
of a pure tone sound source in an unfamiliar environment can be relatively unreliable. We suggest that this error in spatial localisation of pure tones may very well have contributed to the cost in performance in the haptic domain, since the reference frame provided by these sounds is too unreliable to precisely encode the locations of the objects.

\section{Experiment 3}

Apart from the unreliable localisation of auditory tones, it is also possible that the tones were simply distracting during the encoding of the haptic object locations (Driver and Spence, 1998; Farah et al., 1989; Spence et al., 2001) or during the retrieval of the spatial positions of the objects from haptic memory (Hecker and Mapperson, 1997). In this experiment, we directly examined whether the presence of the auditory tones affected the encoding or recall of the haptic scene. To that end, we replicated Experiment 2 but presented the tones during either the learning or test phase only. If non-informative sound disrupts the encoding of the haptic scene then we expected a cost in performance when the tones were presented during the learning component of the trial only compared to tones presented during the test component. Alternatively, if the tones affected the nature of the memory representation then we expected a cost in performance when these tones were presented during the recognition test only (i.e. at the retrieval stage only).

\subsection{Method}

\subsubsection{Participants}

Twenty-four students from Trinity College Dublin (female $=17$, male $=7$ ) participated in this experiment for research credits or pay. Participants were aged between 18 and 40 years (mean age $=24.50$ ). All bar one of the participants reported to be right-hand dominant. None of the participants reported any tactile impairment.

\subsubsection{Design and procedure}

The same apparatus and materials used in Experiment 2 were also used here with the exception that the sounds were either presented in the learning period or test period only of each trial. The experiment was based on a two-way, mixed design with presentation session within a trial of the non-informative sounds (learning or test) as a betweensubjects factor and scene rotation (same or rotated) as a within-subjects factor. Participants were randomly allocated to one of the two auditory conditions: 12 participants were presented with non-informative sounds during the learning session only whereas the other 12 were presented with sounds during the testing session only. The procedure was the same as described in the previous experiments.

\subsection{Results}

The percentage correct responses across both non-informative auditory conditions and scene rotations are plotted in Figure 4. A mixed ANOVA was conducted on the percentage correct performance with non-informative auditory session (learning or test) as the between groups factor and scene rotation (same or rotated) as a within-subjects 
factor. We found a significant main effect of non-informative auditory session $[F(1,20)=5.37, p<0.05]$ : participants who were presented with the auditory tone stimuli during the test session only performed significantly worse than those who were presented with the tones during the learning session only. As in the previous experiments, there was a significant main effect of scene rotation $[F(1,20)=5.28, p<0.05]$ with better performance when the scene was not rotated than when it was rotated. There was no interaction between these two factors $[F(1,20)=1.11, p=0.31]$.

To determine whether performance was enhanced by the non-informative sounds during encoding or whether a cost in performance occurred when sounds were presented during the test session, we compared performance in these two conditions to that of participants in the 'no sound' condition in Experiment 1 using independent $t$-tests. There was no significant difference between performance in the 'no sound' condition and when sounds were presented during the learning session only $[t<1$, ns]. However, performance was significantly worse when sounds were presented during the test session than in the 'no sound' condition $[t=2.57, p<0.05]$.

\subsection{Discussion}

Our findings here suggest that, relative to a no sound condition, non-informative audition disrupts the recall of the haptic scene but not the encoding of the scene. Moreover, we failed to find evidence that the spatial information provided by auditory information enhanced the encoding or retrieval from memory of the positions of the objects through touch. This finding is in contrast to the benefit reported for non-informative vision in tactile perception (e.g. Newport et al., 2002; Pasqualotto et al., submitted). In fact, here we found that during the relatively difficult task of recognising the locations of objects in a scene, auditory sounds incurred a cost in performance.

One possibility for this cost is that participants were distracted by the sounds or that attention was drawn towards the direction of the sound sources rather than the tactile scene during retrieval. However, these explanations do not account for the fact that noninformative sounds disrupted performance only during recall and not during encoding as we would expect a similar attentional effect across both conditions. Instead, the findings from previous studies that spatial information from a non-informative modality has a greater effect on more difficult spatial tasks seem to offer a more likely reason for our results (Press et al., 2004). For example, the cognitive effort involved in recognising which objects had moved position between learning and test may have rendered performance more vulnerable to the effects of non-informative sounds. Furthermore, the fact that localisation of these sounds was relatively uncertain (as determined in the previous experiment) probably contributed to the cost in performance rather than any benefits, as it is typically found with non-informative visual information on tactile spatial perception.

Again, as in the previous experiment, there was no interaction between when the sounds were presented (learning or test) and scene rotation. We suggest that the presence of sounds contributed to a relative uncertainty in the haptic representation in memory, but not to the overall nature of the representation. The effect of rotation across all conditions suggests that haptic scenes are stored in memory as egocentric representations (see Newell et al., 2005) as it was previously found for the representations of scenes encoded through vision (e.g. Diwadkar and McNamara, 1997). 
Figure 4 Plot showing the mean percent correct responses for the haptic scene recognition task in Experiment 3

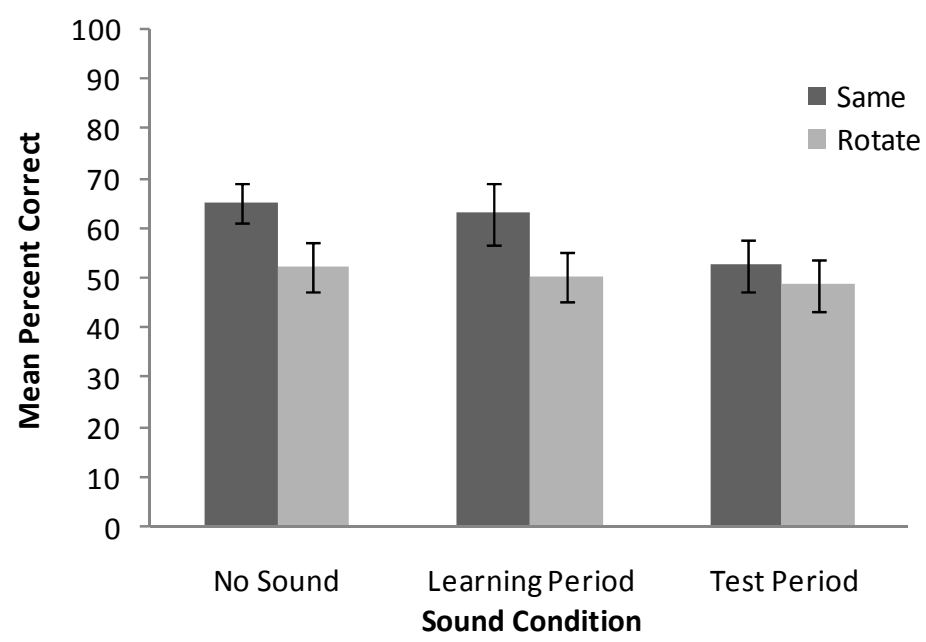

Note: In this experiment, participants received pure tone stimuli in the non-informative sound condition during either the learning session or test session only. The "no sound' condition of Experiment 1 is inserted for comparative purposes. The error bars represent \pm 1 SEM.

\section{Experiment 4}

It remained puzzling to us that an intrinsically spatial modality, such as audition, did not incur the same benefits on haptic performance as the visual modality, even with sound sources known to be relatively easy to localise (such as the white noise bursts in Experiment 1). It is known, e.g. that auditory location cues are used as reliable sources of information for the purposes of spatial navigation (Klatzky et al., 2002, 2003, 2006), especially in the absence of visuo-spatial cues such as in persons who are visually impaired (Thinus-Blanc and Gaunet, 1997).

Previous studies on the role of auditory spatial cues in navigation tasks have generally presented a small number of cues, each separated over time (between 3 and 5 cues; Klatzky et al., 2003). In our experiments described above, when a small number of different auditory stimuli were presented one at a time, a cost in performance was found relative to when the same sounds were presented across locations. As such, our results could either have been affected by the changing spatial locations of different auditory stimuli or to the changes in the auditory frequency themselves. Moreover, localising individual sound positions that are intermittent may be more difficult than localising more stationary sound sources.

In the following experiment, we attempted to assess the role of changing sound sources from the changing sounds themselves on haptic spatial perception by presenting, in any one haptic trial, a single frequency tone from a fixed location. Across trials, different tones were presented to different locations, but the spatial location did not differ within a trial. In other words, we reduced the number of sound locations used in the 
previous experiments (i.e. four) during a haptic trial to one source. Moreover, these sounds were presented during the test phase of the trial only, since the results from the previous experiment indicated no effect of the presence of non-informative sound on haptic encoding. If changing sounds across spatial locations during a trial induced a cost in haptic memory performance, then we expected that a stationary sound source would not incur a cost on haptic performance relative to a 'no sound' condition.

\subsection{Method}

\subsubsection{Participants}

Twenty-four students from Trinity College Dublin (female $=21$, male $=3$ ) participated in this experiment for research credits or pay. Their ages ranged between 18 and 52 years (mean age $=24.50$ ). All participants reported to be right-hand dominant. None of the participants reported any tactile or auditory impairment.

\subsubsection{Auditory stimuli}

The same apparatus and materials used in the previous experiments were again used here. As in Experiments 2 and 3, a specific tone was associated with a specific location; however, in this experiment each tone was presented from a single, stationary location throughout a trial. Based on the results from Experiment 3, tones were presented only during the test phase of each trial.

\subsubsection{Design and procedure}

The experiment was based on a two-way mixed design with location of auditory stimuli as a between-subjects factor (front or rear speaker positions) and scene rotation as the within-subjects factor. Each participant received two blocks of trials during the experiment. In the first block, an auditory stimulus (i.e. a single tone frequency) was presented from a single location for all trials and in the second block, another auditory tone was presented from a different, but consistent location. These locations were pseudo-randomly allocated across participants such that one group was presented with the auditory tone stimuli from only the two front loudspeakers whereas the other group were presented with the stimuli from the two rear loudspeakers (relative to the participant).

There was a total of 16 trials in the experiment with 8 trials within each block. Each block consisted of four trials where the platform was not rotated between the learning and test phase and four trials where the scene was rotated by $60^{\circ}$, all presented in a random order In all other ways, the procedure for the haptic scene recognition task was the same as described in the previous experiments. The experiment took approximately $60 \mathrm{~min}$ for a participant to complete.

\subsection{Results}

We first conducted a two-way, mixed design ANOVA on the correct responses with auditory stimulus location (front or rear loudspeakers) as a between-subjects factor and scene rotation (same or rotated) as a within-subjects factor. There was no main effect of speaker location on performance $[F(1,19)<1$, ns]. The effect of scene rotation approached significance $[F(1,22)=3.39, p=0.08]$ and there was no interaction between these factors. 
Since there was no significant difference between the front and rear loudspeaker locations, the results from these two conditions were collapsed for all subsequent analyses. A separate two-way mixed ANOVA was conducted between the results of this experiment and the results from Experiment 3 when the sounds were presented in the test phase only. Here the effect of scene rotation was significant $[F(1,21)=4.38, p<0.05]$. Interestingly, the difference in performance across the two experiments (when sounds were presented during the test phase only) approached significance $[F(1,21)=3.63$, $p=0.07]$ in that performance was slightly better in Experiment 4 compared to Experiment 3. There was no evidence of an interaction between these two factors $[F(1,21)<1, \mathrm{~ns}]$.

We then conducted a two-way mixed ANOVA on the correct responses with auditory sound locations (single location or 'no sound' condition from Experiment 1) as a between-subjects factor and scene rotation as a within-subjects factor. Once again, there was a significant main effect of rotation $[F(1,30)=6.49, p<0.05]$. There was no significant difference between the sound and no sound conditions $[F(1,30)=1.63$, $p=0.21$, suggesting that presenting a sound from a single source during a trial did not affect performance. There was no evidence of an interaction between these two factors $[F(1,30)<1$, ns $]$ (Figure 5).

\subsection{Discussion}

The primary difference between this experiment and Experiment 3 was the presentation of a single tone for the duration of the test phase in the current experiment compared to the presentation of multiple tones from several locations in Experiment 3. Overall, however, the same sound sources and locations were tested across both experiments. In this experiment, we expected that a sound stimulus consistently presented from a single location during a trial would not reduce haptic spatial performance, if multiple changing sound sources presented during a single trial interfered with this performance (as we speculated in Experiment 3). Although performance in Experiment 4 (overall mean: $52.4 \%$ ) was slightly better than performance in the test phase only condition of Experiment 3 (overall mean: 47.95\%), this difference approached but failed to reach statistical significance. However, haptic performance when just one sound source was present did not differ from performance when no sound was presented (i.e. Experiment 1), in contrast to the cost found between conditions when multiple sounds were presented relative to no sounds. Therefore, it seems that it was the changing spatial locations rather than the tones themselves that affected performance. In other words, these results indicate that auditory spatial information disrupts spatial recognition of haptic scenes relative to no sound and that this cost is unlikely to be attributed to the nature of the changing sound tones across multiple spatial locations.

As found in all the experiments reported here, participants found it more difficult to perform the haptic spatial task when the scene was rotated by $60^{\circ}$. Furthermore, we found no difference between presenting a single tone from the front or rear loudspeakers on scene rotation, although previous studies have suggested that sound localisation is more difficult when stimuli are presented behind the listener than in front of the listener (Makous and Middlebrooks, 1990). We are unclear why sound location did not differ as suggested by previous literature, although we suspect that the difficulty of our task may have obscured any differences between the locations of the sounds. 
Figure 5 Plot showing the mean percent correct responses for the haptic scene recognition task in Experiment 4

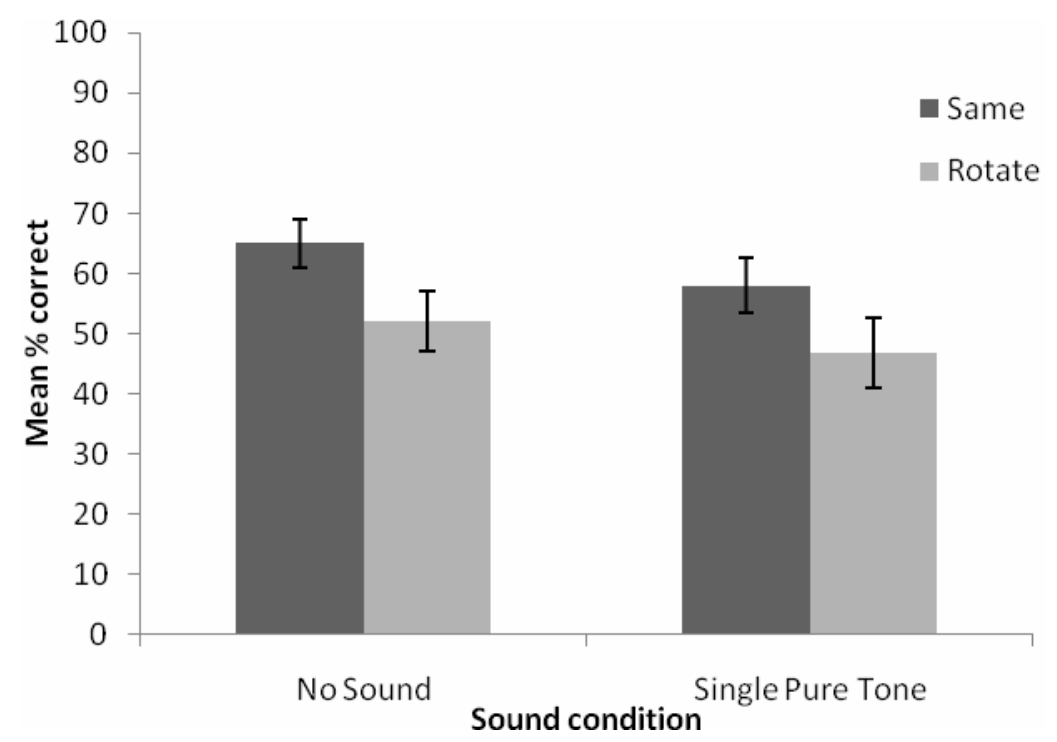

Note: In any one trial, participants received a single pure tone from a fixed auditory source during the test period only. Performance from the 'no sound' condition of Experiment 1 is inserted for comparative purposes. The error bars represent \pm 1 SEM.

\section{Experiment 5}

The pure tones used in Experiment 2 not only may have distracted attention away from the haptic scene, but may also have incurred a cost on performance due to their unfamiliarity. In the following experiment, we further tested whether auditory stimuli can affect performance in a haptic spatial task by presenting more familiar sounds from each of the four loudspeakers. Familiar sounds may incur a benefit on performance since they are thought to be easier to localise compared to unfamiliar stimuli (Mershon, 1997; Mershon et al., 1981; Zahorik and Wightman, 2001).

\subsection{Methods}

\subsubsection{Participants}

Sixteen participants ( 9 females) were between the ages of 20 and 40 years $($ mean $=26.71$ years $)$. All participants were right-handed and reported to have no tactile or hearing impairments and have normal or corrected-to-normal vision.

\subsubsection{Auditory stimuli}

The same apparatus and materials used in the previous experiments were again used here. However, instead of a specific tone associated with a specific location, each location was 
associated with a specific familiar sound (rattle, bubbles in water, alarm clock and running water). These sounds were chosen on the basis that they were associated with stationary objects in real life (i.e. no animal sounds were used), were familiar to participants, the sound could be sustained for the required duration during a trial and each sound was distinct from the other auditory stimuli (semantically as well as physically). Based on the results from Experiment 3, the sounds were presented only during the test phase of each trial.

\subsubsection{Design and Procedure}

The experiment was again based on a mixed design with audition (non-informative sounds or no sounds) as the between-subjects factor and scene rotation as the withinsubjects factor. Participants in this experiment were all allocated to the 'sound' condition only and we compared their performance to the participants allocated to the 'no sound' condition in Experiment 1. In all other respects, the design and procedure were the same as described in Experiments 1 and 3. Each sound was consistently emitted from one location only throughout the experiment.

\subsection{Results}

We compared performance in the familiar sound condition in the present experiment to the 'no-sound' condition in Experiment 1. Therefore, we conducted a mixed-design ANOVA with sound familiarity (familiar sounds vs. no sound) as the between-subjects factor and scene rotation (same vs. rotated) as the within-subjects factor. As in the previous experiments, there was a main effect of scene rotation $[F(1,36)=15.95$, $p=0.0003]$ with better performance for the same $(65.10 \%)$ than rotated $(49.05 \%)$ scenes. There was no main effect of sounds $[F(1,36)<1$, ns] nor an interaction between these factors.

To determine whether the familiarity itself affected performance we compared the results with Experiment 2 in a mixed ANOVA with sound familiarity (familiar vs. unfamiliar) as the between-subjects factor and scene rotation (same vs. rotated) as the within-subjects factor. Although performance to the familiar sounds $(55.04 \%)$ was better than that to the unfamiliar sounds $(46.46 \%)$ this difference failed to reach significance $[F(1,30)=1.8, p=0.19]$. The effect of scene rotation was significant $[F(1,30)=33.56$, $p<0.0001]$. There was no interaction between these two factors.

\subsection{Discussion}

In this experiment, unlike the pure tones in Experiment 2, we failed to find evidence that familiar sounds affected haptic scene perception compared to no sound. Moreover, although performance in the familiar sound condition was better than in the unfamiliar sound condition in Experiment 2, this difference failed to reach statistical significance suggesting that knowledge of the sound is not an important factor in influencing haptic spatial memory. In other words, the enhanced spatial precision which familiar sounds offer relative to unfamiliar sounds (Mershon, 1997) did not affect haptic spatial perception. Once again, performance was significantly worse when the haptic scene was rotated between the learning and testing phase suggesting that the nature of the haptic representation was not affected by the presence or absence of sounds in the environment. 


\section{General discussion}

Previous research investigating the role of non-informative, cross-modal information on spatial performance has typically focused on visual information affecting a haptic task. Given that audition is also an intrinsically spatial modality, we assessed the role of non-informative auditory information on haptic performance. Here we found that, unlike the influence of non-informative vision on haptic performance, non-informative auditory information either had no effect or it disrupted haptic scene recognition relative to an absence of auditory spatial cues, but only under limited circumstances. Specifically, we found evidence that many sound types including white noise or familiar sounds have no effect on haptic spatial performance but that unfamiliar, non-informative auditory stimuli involving pure tones disrupt performance in a haptic scene recognition task.

The experiments presented here involved the haptic exploration of object locations presented in peripersonal space. Thus the spatial localisation of the objects in our tasks was not relative to somatosensory stimulation of the body (such as in Kennett et al., 2001; Newport et al., 2002; Press et al., 2004) but rather to the relative position of objects either to the participant, to each other or to an external reference frame (e.g. Zuidhoek et al., 2004). This type of exploration is arguably less accurate than the two-point threshold tasks based on body representations, since it is thought that the representation of external haptic space is non-Euclidean (Kappers, 1999; Kappers and Koenderink, 1999). We reasoned that since sounds can be reliably localised (Klatzky et al., 2003; Makous and Middlebrooks, 1990) spatial cues from audition would enhance haptic spatial perception.

Localised white noise bursts did not affect haptic scene recognition compared to the absence of any sounds (i.e. the 'no sound' condition). Although we found that localising white noise was relatively more accurate than localising pure tone stimuli, which is consistent with previous literature (Blauert, 1997; Middlebrooks and Green, 1991), we did not find that this relative reliability in external spatial information benefited haptic performance. Since the same white noise bursts were presented at successive locations, without a time interval between presentations, it remains possible that these sounds may have been perceived as a single 'moving' stimulus. If this was the case then localising each single white noise sound source would have been a relatively more difficult task. To alleviate this concern, different pure tones replaced the white noise stimuli in Experiment 2 with each tone corresponding to a distinct spatial location. Instead of these tones enhancing haptic spatial perception, as it was expected (Kennett et al., 2001; Newport et al., 2002), the results of Experiment 2 found that pure tones that are each associated with a particular spatial location can disrupt haptic performance (cf. Press et al., 2004). The results of Experiment 3 revealed that this disruption occurred only during the recall of the haptic scene and not the encoding. In light of the results of Experiment 1, our findings in Experiment 4 also suggest that it was the combination of changes in tones and changes in spatial location that was disruptive, especially during the recall of the haptic scene. Moreover, this finding was not due to the unfamiliar auditory stimuli used in Experiments 3 and 4, since there was no specific task-dependent benefit for familiar sounds used in Experiment 5, but more likely due to the difficultly in localising pure tones.

It is also interesting to note that the nature of the representation of the haptic scene was independent of any influence from the auditory spatial cues. In all experiments, we 
found a robust effect of scene orientation on performance with better performance when the scene was presented in the same orientation during learning and test than when it was rotated. Effects of scene rotation are generally argued to be evidence for egocentric representations of scenes (e.g. Diwadkar and McNamara, 1997; Newell et al., 2005; Simons and Wang, 1998; Wang and Simons, 1999). Furthermore, even when a cost in overall performance was associated with the presence of auditory spatial cues, the effect of scene rotation was not changed. This finding suggests that haptic scenes are represented in memory as egocentric representations (see Newell et al., 2005) but that this overall representation is compromised when relatively unreliable auditory information provides the extrinsic spatial reference frame.

Although our results clearly suggest cross-modal influences from non-informative auditory spatial information on haptic spatial performance, it is not known how these effects occur. It is possible, e.g. that auditory and haptic information activate a common 'where' stream, thus resulting in these multisensory influences on performance. For example, recent neuroimaging studies have also suggested that the functional division of 'what' and 'where' processing in the primate auditory system is similar to that in the visual system (Poremba et al., 2003; Rauschecker and Tian, 2000). Moreover, Poremba et al. reported that areas within these functional 'what' and 'where' streams in the auditory domain overlap with their corresponding visual areas. The findings from a recent study on 'what' and 'where' processing in the visual and tactile domains (see Chan and Newell, 2008) suggest that these overlapping areas may result in cross-modal influences at the behavioural level. These studies suggest that the division between the modalities for the purpose of object and spatial processing may not be as clear cut as previously thought, and that the likelihood of multisensory information influencing a task within a single modality is to be expected.

In our tasks here, participants were instructed to ignore the auditory spatial information therefore it is not clear to what extent unattended information from another modality can nevertheless influence task processing in the attended modality. Recent studies have found that the processing of unattended sounds seems to activate cortical areas which are not normally associated with the more posterior 'dorsal' and 'ventral' processing streams involved in spatial and object processing, respectively (Doeller et al., 2003; Deouell et al., 2007). Therefore, the extent to which unattended auditory spatial information can influence performance in a tactile spatial task, given these differences in cortical areas of activation, is unclear. On the other hand, Poirer et al. (2005) found that moving auditory tones activate cortical areas within the visual dorsal stream normally associated with visuo-spatial processing. As in our Experiment 2, some of the stimuli used by Poirer et al. (2005) varied in frequency. If we assume that haptic spatial processing activates cortical areas which are usually thought of as visual, then the results of Poirer et al. suggest a mechanism for how auditory tones which vary in spatial location can affect spatial processing in the tactile domain.

One could also argue that the effects demonstrated in Experiments 2 and 3 were simply due to divided attention across the haptic and auditory modalities (Driver and Spence, 1998; Farah et al., 1989; Spence et al., 2001). However, it is unlikely that this is the reason for the cost in performance in Experiment 2, since divided attention should affect scene recognition performance in both the learning and the test phase (see Experiment 3). Furthermore, in a debriefing session, participants reported that they found it easy to ignore the auditory tones during the experiment. 
Press et al. (2004) found that irrelevant visual information can either aid or disrupt performance in a tactile spatial task depending on whether the tactile task was difficult or easy, respectively. Our results are in contrast to the finding of Press et al. since our task was relatively difficult, with a range in accuracy of between $55 \%$ and $60 \%$ across all experiments. In our experiments, task difficulty was associated with a disruption in performance by simultaneous auditory spatial information. Thus it is unclear from our data whether increasing task demands would modulate the effect of non-informative sound. This is, however, clearly an area for further research.

Our findings add to the growing body of evidence demonstrating that non-informative information, regardless of modality, can affect performance on a given task. Consequently, it is not enough to simply control for within-modal experimental variables that may affect performance outcomes since spatial information from other modalities, such as changes in equipment location as well as changes in ambient sounds, may have an unpredictable effect on spatial performance in the modality of interest. In sum, our data here suggest that like visuo-spatial information, auditory information that has a spatial component can affect spatial perception in touch. Together with the literature on non-informative vision, these findings suggest that spatial perception is multisensory and that information from both vision and audition, even when irrelevant to the task, can have an effect on performance in the haptic modality.

\section{Acknowledgements}

This research was funded by a post-doctoral fellowship from the Irish Research Council for the Humanities and Social Sciences awarded to JSC and by Science Foundation Ireland, Grant No. 06/IN.1/196.

\section{References}

Arnott, S.R. and Goodale, M.A. (2006) 'Distorting visual space with sound', Vision Research, Vol. 46, pp.1553-1558.

Blauert, J. (1968) 'Ein Beitrag zur Theorie des Vorwärts-Rückwärts-Eindruckes beim Hören [A contribution to the theory of the front-back impression in hearing]', Paper presented at the Proceedings of the 6th International Congress on Acoustics, Tokyo, Japan.

Blauert, J. (1997) Spatial Hearing. London, England: MIT Press.

Caclin, A., Soto-Faraco, S., Kingstone, A. and Spence, C. (2002) 'Tactile "capture" of audition', Perception and Psychophysics, Vol. 64, No. 4, pp.616-630.

Chan, J.S. and Newell, F.N. (2008) 'Behavioural evidence for task-dependent, 'what' versus 'where' processing within and across modalities', Perception and Psychophysics, Vol. 70, No. 1, pp.36-49.

Colavita, F.B. (1974) 'Human sensory dominance', Perception and Psychophysics, Vol. 16, No. 2, pp.409-412.

Deouell, L.Y., Heller, A.S., Malach, R., D’Esposito, M. and Knight, R.T. (2007) 'Cerebral responses to change in spatial location of unattended sounds', Neuron, Vol. 55, pp.985-996.

Diwadkar, V.A. and McNamara, T.P. (1997) 'Viewpoint dependence in scene recognition', Psychological Science, Vol. 8, pp.302-307. 
Doeller, C.F., Opitz, B., Mecklinger, A., Krick, C., Reith, W. and Schroeger, E. (2003) 'Prefrontal cortex involvement in preattentive auditory deviance detection: neuroimaging and electrophysiological evidence', NeuroImage, Vol. 20, No. 2, pp.1270-1282.

Driver, J. and Grossenbacher, P.G. (1996) 'Multimodal spatial constraints on tactile selective attention', in J.L. McClelland (Ed.), Attention and Performance XVI: Information Integration in Perception and Communication. MA, USA: The MIT Press, pp.209-235.

Driver, J. and Spence, C. (1998) 'Crossmodal attention', Current Opinion in Neurobiology, Vol. 8, No. 2, pp.245-253.

Farah, M.J., Wong, A.B., Monheit, M.A. and Morrow, L.A. (1989) 'Parietal lobe mechanisms of spatial attention: modality-specific or supramodal?' Neuropsychologia, Vol. 27, No. 4, pp.461-470.

Graziano, M.S.A. and Gross, C.G. (1993) A bimodal map of space: somatosensory receptive fields in the macaque putamen with corresponding visual receptive fields', Experimental Brain Research, Vol. 97, pp.97-109.

Haggard, P., Christakou, A. and Serino, A. (2007) 'Viewing the body modulates tactile receptive fields', Experimental Brain Research, Vol. 180, pp.187-193.

Hecker, R. and Mapperson, B. (1997) 'Dissociation of visual and spatial processing in working memory', Neuropsychologia, Vol. 35, No. 5, pp.599-603.

Holmes, N.P. and Spence, C. (2004) 'The body schema and multisensory representation(s) of peripersonal space', Cognitive Processing, Vol. 5, pp.94-105.

Jeffress, L.A. (1972) 'Binaural signal detection: vector theory', in J.V. Tobias (Ed.), Foundations of Modern auditory Theory, Vol. 2, New York: Academic, pp.349-368.

Kappers, A.M.L. (1999) 'Large systematic deviations in the haptic perception of parellelity', Perception, Vol. 28, No. 8, pp.1001-1012.

Kappers, A.M.L. and Koenderink, J.J. (1999) 'Haptic perception of spatial relations', Perception, Vol. 28, No. 6, pp.781-795.

Kennett, S., Taylor-Clarke, M. and Haggard, P. (2001) 'Noninformative vision improves the spatial resolution of touch in humans', Current Biology, Vol. 11, No. 15, pp.1188-1191.

Klatzky, R.J., Lippa, Y., Loomis, J.M. and Golledge, R.G. (2002) 'Learning directions of objects specified by vision, spatial audition, or auditory spatial language', Learning and Memory, Vol. 9, pp.364-367.

Klatzky, R.J., Lippa, Y., Loomis, J.M. and Golledge, R.G. (2003) 'Encoding, learning, and spatial updating of multiple object locations specified by 3-D sound, spatial language, and vision', Experimental Brain Research, Vol. 149, pp.48-61.

Klatzky, R.J., Marston, J.R., Giudice, N.A., Golledge, R.G. and Loomis, J.M. (2006) 'Cognitive load of navigating without vision when guided by virtual sound versus spatial language', Journal of Experimental Psychology: Applied, Vol. 12, No. 4, pp.223-232.

Lavie, N. (1995) 'Perceptual load as a necessary condition for selective attention', Journal of Experimental Psychology: Human Perception and Performance, Vol. 21, No. 3, pp.451-468.

Lavie, N. and Tsal, Y. (1994) 'Perceptual load as a major determinant of the locus of selection in visual attention', Perception and Psychophysics, Vol. 56, No. 2, pp.183-197.

Lloyd, D.M., Merat, N., McGlone, F. and Spence, C. (2003) 'Crossmodal links between audition and touch in covert endogenous spatial attention', Perception and Psychophysics, Vol. 65, No. 6, pp.901-924.

Loomis, J.M., Klatzky, R.L., Philbeck, J.W. and Golledge, R.G. (1998) 'Assessing auditory distance perception using perceptually directed action', Perception and Psychophysics, Vol. 60, No. 6, pp.966-980.

Makous, J.C. and Middlebrooks, J.C. (1990) 'Two-dimensional sound localization by human listeners', Journal of the Acoustical Society of America, Vol. 87, No. 5, pp.2188-2200. 
Mershon, D.H. (1997) 'Phenomenal geometry and the measurement of perceived auditory distance', in R.H. Gilkey and T.R. Anderson (Eds.), Binaural and Spatial Hearing in Real and Virtual Environments. Hillsdale, NJ: Erlbaum, pp.257-274.

Mershon, D.H., Desaulniers, D.H., Kiefer, S.A., Amerson, T.L.J. and Mills, J.T. (1981) 'Perceived loudness and visually-determined auditory distance', Perception, Vol. 10, No. 5, pp.531-543.

Middlebrooks, J.C. and Green, D.M. (1991) 'Sound localization by human listeners', Annual Review of Psychology, Vol. 42, pp.135-159.

Newell, F.N., Woods, A.T., Mernagh, M. and Bülthoff, H.H. (2005) 'Visual, haptic and crossmodal recognition of scenes', Experimental Brain Research, Vol. 161, pp.233-242.

Newport, R., Rabb, B. and Jackson, S.R. (2002) 'Noninformative vision improves haptic spatial perception', Current Biology, Vol. 12, No. 19, pp.1661-1664.

Pasqualotto, A., Finucane, C. and Newell, F.N. (submitted) 'Non-informative visuospatial information improves memory for haptic scenes'.

Pasqualotto, A., Finucane, C.M. and Newell, F.N. (2005) 'Visual and haptic representations of scenes are updated with observer movement', Experimental Brain Research, Vol. 166, pp.481-488.

Pears, S. and Jackson, S.R. (2004) 'Cognitive neuroscience: vision and touch are constant companions', Current Biology, Vol. 14, pp.R349-R350.

Poirer, C., et al. (2005) 'Specific activation of the V5 brain area by auditory motion processing: an fMRI study', Cognitive Brain Research, Vol. 25, pp.650-658.

Poremba, A., Saunders, R.C., Crane, A.M., Cook, M., Sokoloff, L. and Mishkin, M. (2003) 'Functional mapping of the primate auditory system', Science, Vol. 299, No. 5606, pp.568-572.

Press, C., Taylor-Clarke, M., Kennett, S. and Haggard, P. (2004) 'Visual enhancement of touch in spatial body representation', Experimental Brain Research, Vol. 154, pp.238-245.

Rauschecker, J.P. and Tian, B. (2000) 'Mechanisms and streams for processing of "what" and "where", in auditory cortex', Proceedings of the National Academy of Science, Vol. 97, No. 22, pp.11800-11806.

Rizzolatti, G., Fadiga, L., Fogassi, L. and Gallese, V. (1997) 'The space around us', Science, Vol. 277, No. 5323, pp.190-191.

Schicke, T. (2007) 'Human peripersonal space: evidence from functional magnetic resonance imaging', Journal of Neuroscience, Vol. 27, No. 14, pp.3616-3617.

Simons, D.J. and Wang, R.F. (1998) 'Perceiving real-world viewpoint changes', Psychological Science, Vol. 9, No. 4, pp.315-320.

Simons, D.J., Wang, R.F. and Roddenberry, D. (2002) 'Object recognition is mediated by extraretinal information', Perception and Psychophysics, Vol. 64, No. 4, pp.521-530.

Spence, C. and Driver, J. (1996) 'Audiovisual links in endogenous covert spatial attention', Journal of Experimental Psychology: Human Perception and Performance, Vol. 22, No. 4, pp.1005-1030.

Spence, C., Nicholls, M.E.R. and Driver, J. (2001) 'The cost of expecting events in the wrong sensory modality', Perception and Psychophysics, Vol. 63, No. 2, pp.330-336.

Taylor-Clarke, M., Kennett, S. and Haggard, P. (2002) 'Vision modulates somatosensory cortical processing', Current Biology, Vol. 12, pp.233-236.

Thinus-Blanc, C. and Gaunet, F. (1997) 'Representation of space in blind persons: Vision as a spatial sense?' Psychological Bulletin, Vol. 121, No. 1, pp.20-42.

Trahiotis, C. and Stern, R.M. (1989) 'Lateralization of bands of noise: effects of bandwidth and differences of interaural time and phase', Journal of the Acoustical Society of America, Vol. 86, No. 4, pp.1285-1293. 
Wang, R.F. and Simons, D.J. (1999) 'Active and passive scene recognition across views', Cognition, Vol. 70, pp.191-210.

Zahorik, P.A. and Wightman, F.L. (2001) 'Loudness constancy with varying sound source distance', Nature Neuroscience, Vol. 4, No. 1, pp.78-83.

Zuidhoek, S., Visser, A., Bredero, M.E. and Postma, A. (2004) 'Multisensory integration mechanisms in haptic space perception', Experimental Brain Research, Vol. 157, No. 2, pp.265-268. 